\title{
Pentoxifylline and prevention of hyperoxia-induced lung injury in neonatal rats
}

\author{
Beatriz Almario', Shu $\mathrm{Wu}^{1}$, Jinghong Peng ${ }^{1}$, Deepthi Alapati', Shaoyi Chen ${ }^{1}$ and Ilene R.S. Sosenko ${ }^{1}$
}

INTRODUCTION: Oxygen exposure plays an important role in the pathogenesis of bronchopulmonary dysplasia (BPD). The phosphodiesterase inhibitor pentoxifylline (PTX) has antiinflammatory and antifibrotic effects in multiple organs. It was hypothesized that PTX would have a protective effect on hyperoxia-induced lung injury (HILI).

METHODS: Newborn Sprague-Dawley rats were exposed to >95\% oxygen $\left(\mathrm{O}_{2}\right)$ and injected subcutaneously with normal saline (NS) or PTX $(75 \mathrm{mg} / \mathrm{kg})$ twice a day for $9 \mathrm{~d}$. NS-injected, room air-exposed pups were controls. At days 4 and 9, lung tissue was collected to assess edema, antioxidant enzyme (AOE) activities, and vascular endothelial growth factor (VEGF) expression. At day 9, pulmonary macrophage infiltration, vascularization, and alveolarization were also examined.

RESULTS: At day 9, treatment with PTX significantly increased survival from 54\% to $88 \%$ during hyperoxia. Treatment with PTX significantly decreased lung edema and macrophage infiltration. PTX treatment increased lung AOE activities including those of superoxide dismutase (SOD), catalase (CAT), and glutathione peroxidase (GPX). Furthermore, PTX treatment also increased the gene expression of VEGF189 and VEGF165, increased VEGF protein expression, and improved pulmonary vascularization.

DISCUSSION: These data indicate that the reduced lung edema and inflammation, increased AOE activities, and improved vascularization may be responsible for the improved survival with PTX during hyperoxia. PTX may be a potential therapy in reducing some of the features of BPD in preterm newborns.

D espite improvements in neonatal intensive care, bronchopulmonary dysplasia (BPD), the chronic lung disease of premature infants, is a major cause of long-term hospitalization, recurrent respiratory illnesses, and mortality. The etiology of BPD is complex and has been linked to oxidative stress, mechanical ventilation, infection, and inflammation, as well as genetic susceptibility (1). The pathogenesis of BPD is poorly understood and no effective therapy has yet been developed.

Oxygen toxicity in the developing lung is well known for its contribution to the pathogenesis of BPD. Evidence suggests that one important mechanism involved in lung injury during hyperoxia is direct oxidative damage through increased production of reactive oxygen species. The pulmonary antioxidant enzyme (AOE) system, specifically superoxide dismutase (SOD), catalase (CAT), and glutathione peroxidase (GPX), is a protective mechanism that confers resistance and minimizes toxicity from hyperoxia-induced reactive oxygen species (2-4). However, even though newborn animals show marked tolerance to hyperoxia as compared with adults $(5,6)$, premature newborns have deficient endogenous AOE activities (7) and limited capacity to augment their levels during oxygen exposure (8). Induction or replacement of AOE activity may be part of the therapeutic approach to minimizing reactive oxygen species damage during hyperoxia.

Evidence has demonstrated that downregulation of vascular endothelial growth factor (VEGF) expression during hyperoxia is another important mechanism involved in lung injury and BPD (9). VEGF promotes vessel growth and remodeling, improves endothelial survival, and contributes to the maintenance of alveolar structures (10). VEGF A, in particular, is a major regulator of angiogenesis. VEGF121, VEGF165, and VEGF189 represent the predominant isoforms of VEGF A in the lung. They show different expressions and responses during lung development and injury, and their role in pulmonary vascularization is yet to be completely understood $(11,12)$. Treatment with recombinant VEGF has improved alveolarization during hyperoxia (13), suggesting that enhancing VEGF expression may be an important strategy to prevent hyperoxiainduced lung injury (HILI) in neonates.

Pentoxifylline (PTX), a methylxanthine derivative and phosphodiesterase inhibitor, has immunomodulatory and antifibrotic properties. It is proposed to have a therapeutic role in attenuating tissue injury associated with sepsis and shock in animal models and humans, including neonates (14-16). Studies in rabbits have shown increased levels of CAT, SOD, and GPX during ischemia and reperfusion injury after PTX treatment (17). Studies have also shown that PTX is able to attenuate lung injury related to mechanical ventilation and meconium $(18,19)$. In neonatal HILI models, PTX treatment decreased IL-6 concentration in bronchoalveolar lavage (20), reduced alveolar fibrin deposition, and prolonged survival (21). However, whether PTX treatment affects AOE and VEGF expression in neonatal HILI is unknown.

'Department of Pediatrics, Batchelor Children's Research Institute, University of Miami Miller School of Medicine, Miami, Florida. Correspondence: Ilene R.S. Sosenko (Isosenko@med.miami.edu) 
In this study, we hypothesized that PTX administration would improve survival in hyperoxia, which would be associated with enhancement of $\mathrm{AOE}$ activities and upregulation of pulmonary VEGF expression in a newborn rat model of HILI. Our findings suggest a therapeutic potential of PTX in reducing some of the features of BPD in preterm newborns.

\section{RESULTS}

\section{PTX Improves Survival}

Pups exposed to hyperoxia for $9 \mathrm{~d}$ had a significantly increased mortality as compared with those exposed to room air (RA). However, treatment with PTX dramatically improved survival from $54 \%$ to $88 \%$ during hyperoxia $(P<0.001$; Figure 1$)$. Survival between $\mathrm{O}_{2}+$ normal saline (NS) and $\mathrm{O}_{2}+$ PTX groups was similar until day 6. After day 7, mortality in the $\mathrm{O}_{2}$ + PTX group remained stable until the experiment ended on day 9 , whereas the mortality in the $\mathrm{O}_{2}+\mathrm{NS}$ group continued to increase.

\section{PTX Reduces Lung Edema}

By day 4, there was no difference in lung wet-to-dry-weight ratio between animals raised in RA and animals exposed to $\mathrm{O}_{2}$. At day 9, lung wet-to-dry-weight ratio was significantly increased in $\mathrm{O}_{2}$-exposed groups. However, animals treated with PTX showed a significantly lower lung wet-to-dry-weight ratio as compared with the $\mathrm{O}_{2}+$ NS group (Figure 2), suggesting less pulmonary edema in the PTX-treated group.

\section{PTX Decreases Macrophage Infiltration}

At day 9, macrophages were barely detected in RA + NS lungs. In contrast, in $\mathrm{O}_{2}+\mathrm{NS}$ lungs, there were many macrophages in alveolar airspaces, whereas fewer macrophages were detected in $\mathrm{O}_{2}+$ PTX lungs (Figure 3). Quantification of macrophages demonstrated an eightfold increase in $\mathrm{O}_{2}+$ NS lungs as compared with RA + NS lungs. Treatment with PTX produced significantly less macrophage infiltration as

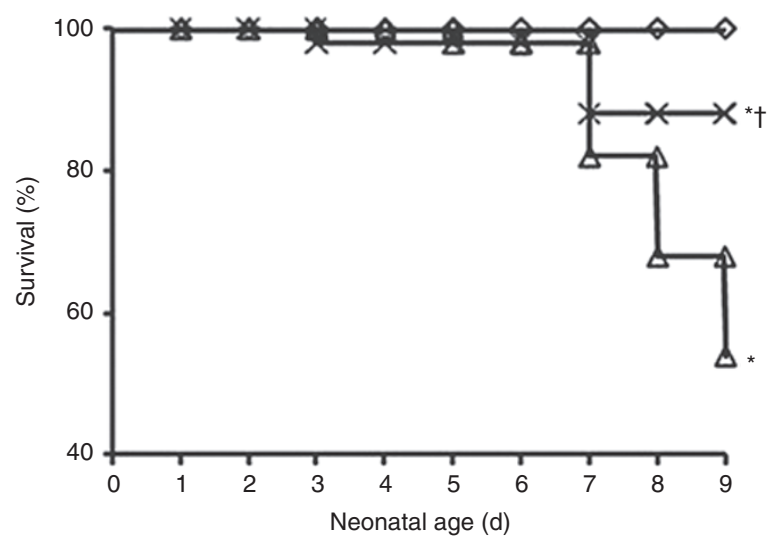

Figure 1. PTX improves survival. Kaplan-Meier survival curve of newborn rats exposed to room air and normal saline (RA + NS; open diamonds), hyperoxia and $\mathrm{NS}\left(\mathrm{O}_{2}+\mathrm{NS}\right.$; open triangles), or hyperoxia and PTX $\left(\mathrm{O}_{2}+\right.$ PTX; crosses). $n=57 /$ group. Data are expressed as percentage of pups surviving at the observed time point. ${ }^{*} P<0.001$, RA group vs. hyperoxia groups. ${ }^{\dagger} P<0.001, \mathrm{O}_{2}+$ PTX vs. $\mathrm{O}_{2}+\mathrm{NS}$. NS, normal saline; $\mathrm{O}_{2^{\prime}}$ oxygen; PTX, pentoxifylline; RA, room air. compared with $\mathrm{O}_{2}+$ NS lungs. These results suggest that PTX is protective against hyperoxia-induced macrophage infiltration.

\section{PTX Enhances AOE Activity}

To assess the effect of hyperoxia and PTX treatment on the pulmonary antioxidant defense system against free radicals, we studied CAT, SOD, and GPX activities. No differences were observed in these enzyme activities among the three study groups at day 4 (Figure 4a). At day 9 , as compared with the RA + NS group, pups in the $\mathrm{O}_{2}+$ PTX group showed significant increases in CAT $(236.2 \pm 59.7$ vs. $416.2 \pm 91.1, P<0.001)$, total SOD $(11,631.4 \pm 4,282.6$ vs. $55,758 \pm 9,579.1, P<0.001)$, and GPX $(0.26 \pm 0.1$ vs. $1.03 \pm 0.4, P<0.001)$ all expressed as activity units/mg DNA (Figure $4 \mathrm{~b}$ ). CAT activity was also significantly elevated in the $\mathrm{O}_{2}+$ PTX group as compared with the $\mathrm{O}_{2}+\mathrm{NS}$ group $(416.2 \pm 91.1$ vs. $292.5 \pm 52.4, P<0.001$; Figure $4 b$ ). These changes were not a result of differences in lung protein or DNA content between the $\mathrm{O}_{2}+$ PTX and $\mathrm{O}_{2}+$ NS groups (data not shown).

\section{PTX Increases VEGF Expression and Improves Vascular Development}

We investigated lung VEGF189, VEGF165, and VEGF121 expression using semiquantitative reverse-transcriptase PCR. There was no difference in the expression of the three isoforms at day 4 among the three study groups (data not shown). In contrast, at day 9, the $\mathrm{O}_{2}+\mathrm{NS}$ group had significantly decreased expression of VEGF189 and VEGF165 as compared with the RA + NS group (VEGF189: $0.31 \pm 0.07$ vs. $0.4 \pm 0.02$, $P<0.05$; VEGF165: $0.29 \pm 0.06$ vs. $0.37 \pm 0.01, P=0.001$ ). However, PTX treatment significantly increased the expression of these two isoforms as compared with $\mathrm{O}_{2}+$ NS pups (VEGF189: $0.61 \pm 0.005$ vs. $0.31 \pm 0.07, P<0.001$; VEGF165: $0.37 \pm 0.02$ vs. $0.29 \pm 0.06, P<0.05$ ) (Figure $5 \mathrm{a}$ ). There was no change in the expression of VEGF121 after hyperoxia or exposure to PTX. To evaluate the correlation between VEGF isoform expression and protein expression, we performed

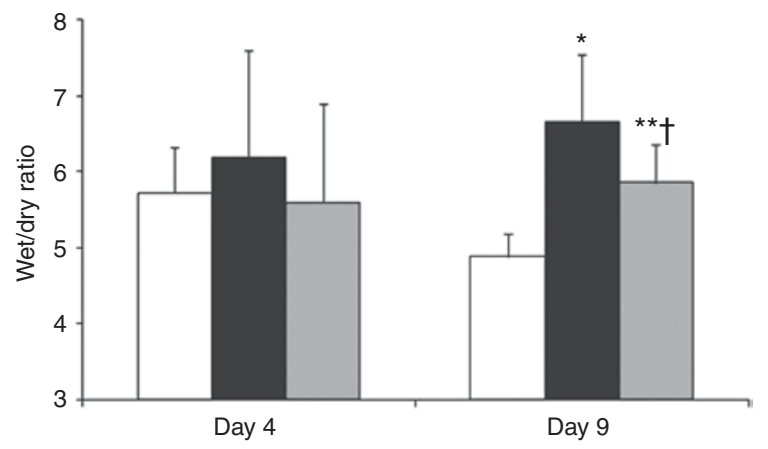

Figure 2. PTX decreases lung edema. At 9 days, hyperoxia increased wet-to-dry-weight ratio in the presence of NS (black bars) or PTX (gray bars) as compared with the RA group (white bars). However, PTX treatment decreased wet-to-dry-weight ratio as compared with NS during hyperoxia. $n=13-14 /$ group, ${ }^{*} P<0.001$ and ${ }^{* *} P<0.01$ as compared with $\mathrm{RA}^{+} P<0.01$ as compared with $\mathrm{O}_{2}+\mathrm{NS}$. NS, normal saline; $\mathrm{O}_{2^{\prime}}$ oxygen; PTX, pentoxifylline; RA, room air. 

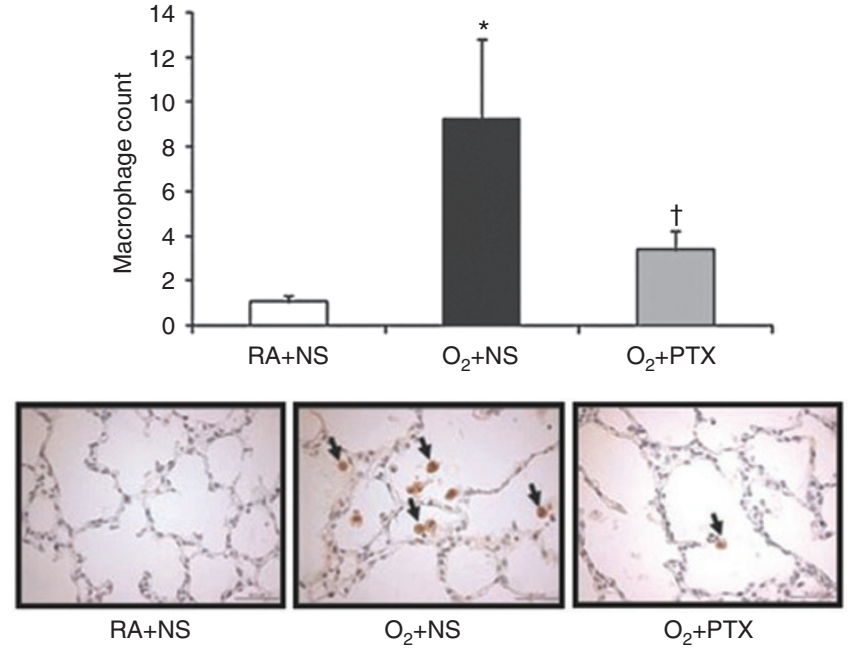

Figure 3. PTX decreases macrophage infiltration. Macrophage infiltration was assessed on Mac3-stained lung tissue sections. Hyperoxia increased macrophage infiltration in airspaces as compared with the RA group, but treatment with PTX attenuated hyperoxia-induced macrophage infiltration. $n=6$ /group. ${ }^{*} P<0.001$ as compared with $\mathrm{RA}+\mathrm{NS} ;{ }^{\dagger} P<0.001$ as compared with $\mathrm{O}_{2}+\mathrm{NS}$. Original magnification: $\times 40$. Bar $=50 \mu \mathrm{m}$. $\mathrm{NS}$, normal saline; $\mathrm{O}_{2}$, oxygen; $\mathrm{PTX}$, pentoxifylline; RA, room air.

enzyme-linked immunosorbent assay at day 9. Although there was no significant decrease in VEGF concentration when comparing the $\mathrm{O}_{2}+\mathrm{NS}$ and the RA + NS groups, treatment with PTX significantly increased VEGF expression in comparison with NS-exposed animals during hyperoxia (106.8 \pm 39.8 vs. $49.8 \pm 41.4, P<0.01$; Figure $5 b$ ).

To determine whether increased VEGF expression by PTX leads to improved vascular development, we assessed vascular density on von Willebrand factor-stained lung tissue sections. The lungs from the $\mathrm{O}_{2}+$ NS group had $65 \%$ decreased vascular density as compared with the normoxia group. In contrast, treatment with PTX significantly increased vascular density during hyperoxia as compared with the $\mathrm{O}_{2}+\mathrm{NS}$ group (4.7 \pm 0.5 vs. $3.4 \pm 0.8, P<0.01$; Figure 5 c).

\section{Effects of PTX on Alveolar Structure and Fibrosis}

To evaluate the effect of PTX treatment on alveolar development, we performed lung histology and morphometry on day 9. On histological examination, lungs in the normoxia group displayed normal alveolarization. In contrast, lungs from hyperoxia groups, both NS and PTX, demonstrated distal airspace enlargement, decreased septation, and a reduction in complexity (data not shown). Morphometric analysis demonstrated a significant decrease in radial alveolar count and a significant increase in mean linear intercept in the $\mathrm{O}_{2}$ groups (as compared with the RA group) and there was no significant difference between the $\mathrm{O}_{2}+\mathrm{NS}$ and $\mathrm{O}_{2}+$ PTX groups (data not shown). We assessed connective tissue growth factor (CTGF) and $\alpha$-smooth muscle actin ( $\alpha$-SMA) expression as markers for fibrosis by western blot analysis on days 4 and 9 . On day 4 , there was no difference in CTGF and $\alpha$-SMA expression among the groups. On day 9, hyperoxia exposure significantly increased
CTGF and a-SMA expression as compared with normoxia exposure, but there was no significant difference between $\mathrm{O}_{2}+$ NS and $\mathrm{O}_{2}+$ PTX groups (data not shown).

\section{DISCUSSION}

Consistent with our hypothesis, we have found that therapy with PTX (a phosphodiesterase inhibitor) during $9 \mathrm{~d}$ of hyperoxia exposure increased AOE activity, increased VEGF expression, improved vascular formation, decreased pulmonary edema and macrophage infiltration, and improved survival in newborn rats. These data highlight some of the potential mechanisms by which PTX protects against neonatal HILI.

The lung's ability to respond to oxidative stress depends largely on its capacity to upregulate protective antioxidants. Newborn experimental animals are more tolerant than adults to hyperoxia. However, preterm experimental animals and presumably newborn infants as well have deficient endogenous AOE activity and limited capacity to augment their levels of protective AOEs during oxygen exposure to overcome oxidative stress $(7,8)$. Studies have established positive correlations between relative resistance to hyperoxia and increase of some or all of the pulmonary AOEs in newborn animals and humans $(22,23)$. Bucher et al. demonstrated a significant increase in GPX and SOD but not in CAT activity in newborn rats exposed to $\mathrm{O}_{2}$ $>95 \%$ for $6 \mathrm{~d}$ and in all three AOEs when exposed for $12 \mathrm{~d}(2)$. Ilizarov et al. demonstrated that overexpression of manganeseSOD improves survival of pulmonary epithelial cells during hyperoxia and CAT offers additional protection when coexpressed with manganese-SOD (24). Treatment with PTX has been shown to increase AOE levels during ischemia and reperfusion injury in different tissues and organs (17); however, no studies have been reported on its effect on AOE levels during prolonged hyperoxia. Although the absolute AOE activities at day 4 were found to actually be higher than on day 9 , we found a significant increase in GPX activity after $9 \mathrm{~d}$ of hyperoxia in control rats, whereas treatment with PTX increased the AOE activities of CAT, SOD, and GPX, indicating that PTX may increase tolerance to hyperoxia by improving cellular antioxidant defense mechanisms. To the best of our knowledge, this is the first study showing a PTX effect on lung AOE activity levels during prolonged hyperoxia.

VEGF plays a central role in normal lung development. Inhibition of angiogenesis reduces alveolarization (25) and VEGF expression is decreased in infants dying of BPD (26). As previously reported (27), we found suppression of VEGF expression in newborn rat lungs during hyperoxia. VEGF is a potent endothelial cell mitogen that stimulates angiogenesis, promotes vessel remodeling, enhances endothelial survival, and maintains alveolar structures. Different isoforms have different affinities for heparin and receptors, and distinct tempo-spatial expression of these isoforms suggests different function. Mice expressing only VEGF121 had impaired lung vascular and airspace formation, indicating an essential role for the heparin sulfate-binding VEGF165 and VEGF189 isoforms in lung development (28). In our study, we demonstrated that PTX protects against hyperoxia-induced 
a
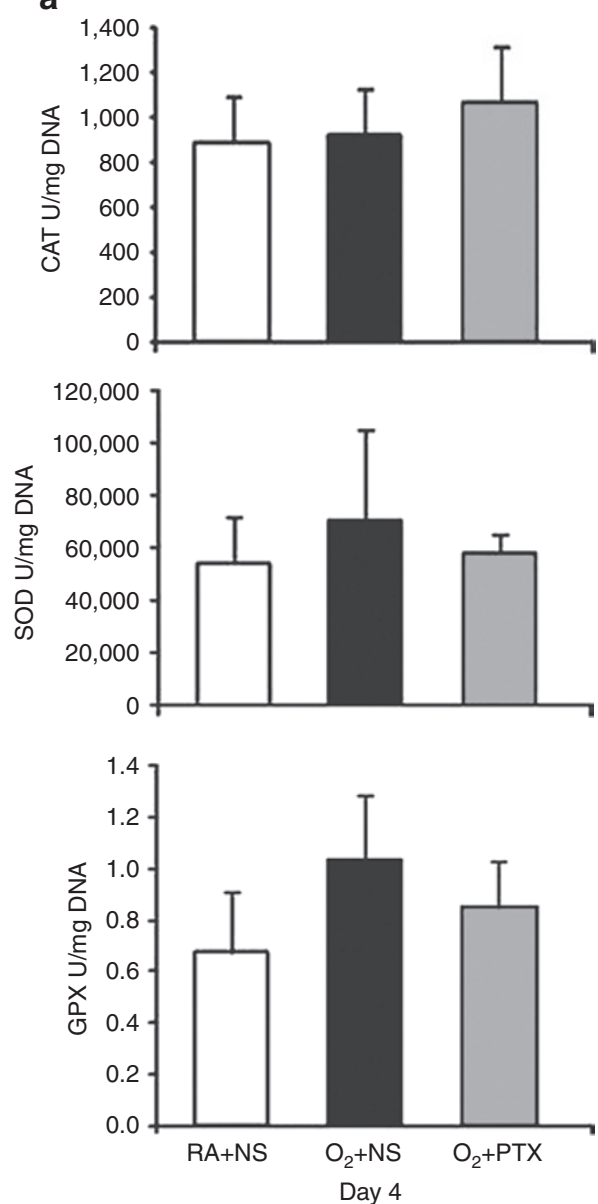

b
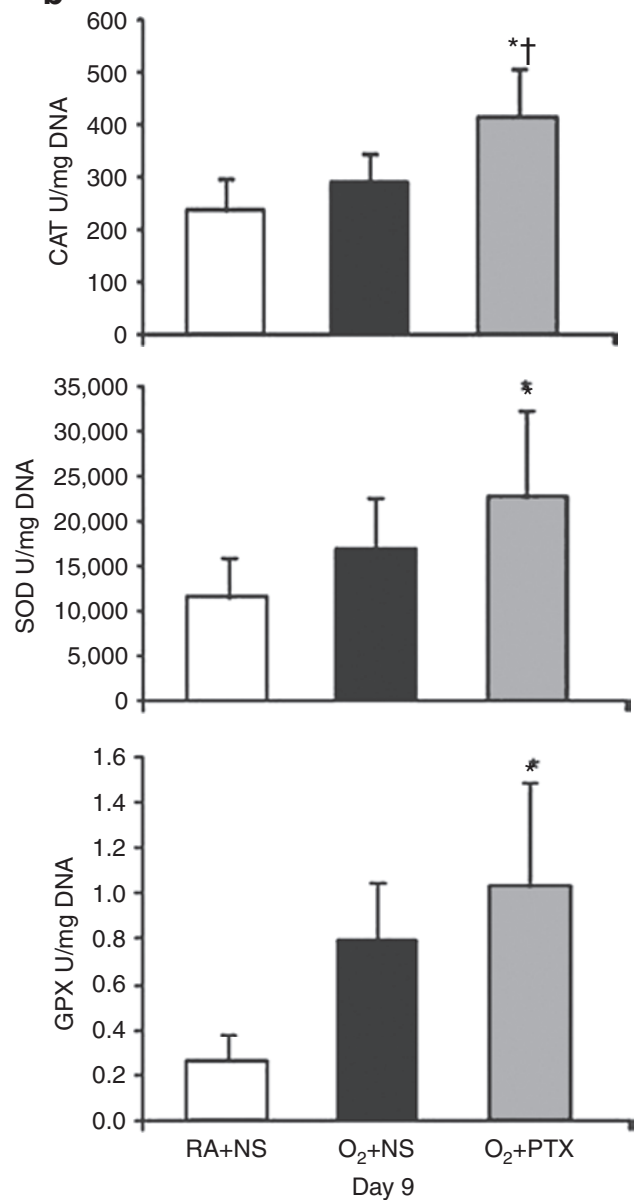

Figure 4. PTX enhances antioxidant enzyme activity. (a) Comparison of CAT, total SOD, and GPX activities at day 4 did not show differences between the three study groups ( $n=7 /$ group). (b) At day 9, as compared with normoxia, hyperoxia alone increased GPX activity and PTX treatment with hyperoxia increased CAT, SOD, and GPX activities. CAT activity was elevated in the $\mathrm{O}_{2}+$ PTX group as compared with the $\mathrm{O}_{2}+$ NS group. $n=12-14 /$ group. ${ }^{*} P<0.001$ as compared with the RA + NS group; ${ }^{\dagger} P<0.001$ as compared with the $\mathrm{O}_{2}+\mathrm{NS}$ group. CAT, catalase; GPX, glutathione peroxidase; NS, normal saline; $\mathrm{O}_{2^{\prime}}$ oxygen; PTX, pentoxifylline; RA, room air; SOD, superoxide dismutase.

downregulation of VEGF189 and VEGF165 isoform expression as well as downregulation of VEGF protein expression. Kunig et al. reported enhanced vascularization in animals treated with VEGF during recovery after hyperoxia (13). Our study demonstrated that PTX treatment during hyperoxia is able to markedly increase pulmonary vascular density, which may be induced by increased VEGF expression. We found that VEGF121 expression was constant in all three study groups, indicating that VEGF121 may not be influenced by oxygen stress as seen in a rabbit model (28). Previous studies have shown that VEGF121 is a predominant form before embryonic day 14, indicating that this isoform has a unique role early in lung development (29), with perhaps a less important role toward birth, leading to a decreased expression and a lack of response during oxygen exposure.

As expected, lung edema was decreased in rat pups treated with PTX during hyperoxia. This decreased lung edema may be associated with increased activity in the AOE system as a result of PTX, which could lead to decreases in reactive oxygen species tissue damage and inflammation, thus resulting in decreased microvascular permeability. In addition, upregulation of VEGF expression by PTX may stabilize the endothelium, preventing further development of pulmonary leakage.

Previous studies have shown that hyperoxia induces fibrotic gene expression such as that of CTGF (30). Furthermore, antiCTGF therapy attenuates hyperoxia-induced alveolar damage and vascular remodeling (31). Multiple studies in kidney and liver have demonstrated that PTX downregulates the expression of CTGF $(32,33)$; therefore, we expected that PTX would decrease CTGF expression and lung fibrosis during hyperoxia. Consistent with previous studies, we found that hyperoxia increases CTGF and $\alpha$-SMA expression. However, we failed to observe downregulation of CTGF and a-SMA expression by PTX. We also did not observe a beneficial effect of PTX on alveolarization during hyperoxia.

Beneficial effects of PTX treatment on survival have been reported in sepsis and in HILI models $(21,34)$. Our study demonstrated survival improvement from 54\% to $88 \%$ with PTX. Our results, showing a positive effect of PTX on 


\section{a}
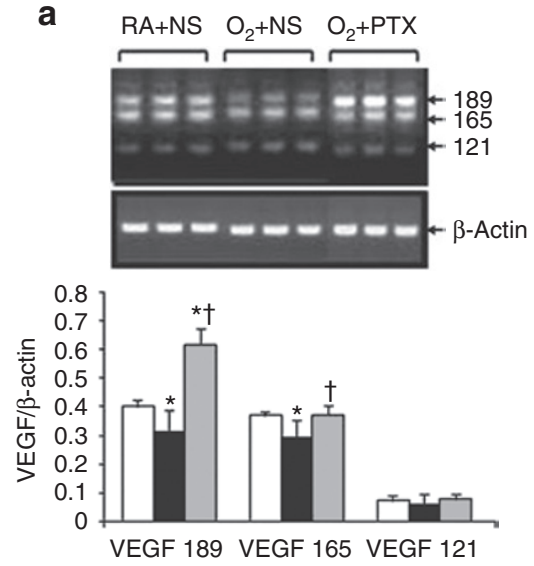

b

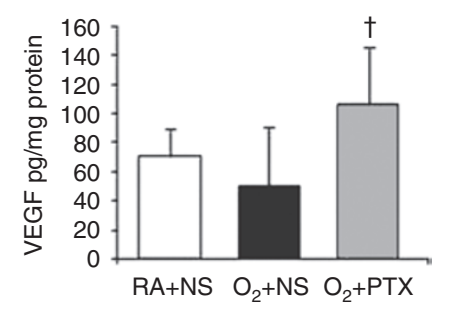

C
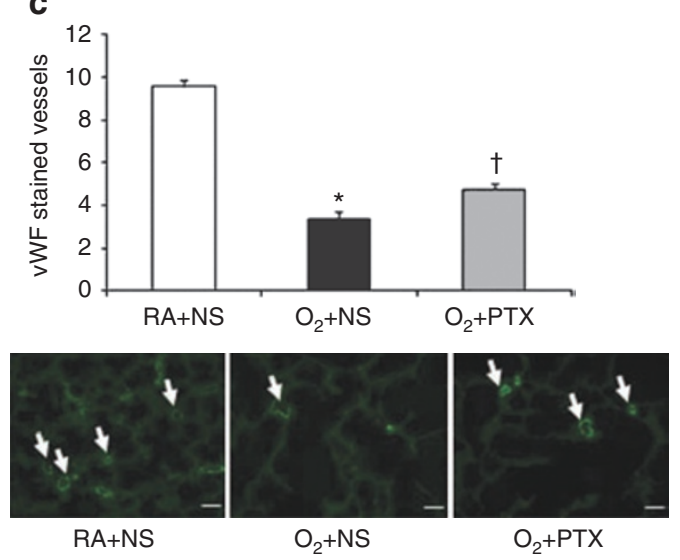

Figure 5. PTX increases VEGF expression and improves vascular development. (a) At day 9, semiquantitative reverse-transcriptase PCR analysis demonstrated a significant decrease of lung VEGF189 and VEGF165 expression in the $\mathrm{O}_{2}+$ NS group as compared with the RA + NS group. PTX treatment significantly increased expression of VEGF189 and VEGF165 as compared with the $\mathrm{O}_{2}+$ NS group. RA + NS group, white bars; $\mathrm{O}_{2}+$ NS group, black bars; $\mathrm{O}_{2}$ + PTX group, gray bars. $n=6 /$ group. ${ }^{*} P<0.001$ as compared with $\mathrm{RA}+\mathrm{NS}$; ${ }^{+} P<0.001$ as compared with $\mathrm{O}_{2}+\mathrm{NS}$. (b) Enzyme-linked immunosorbent assay demonstrated a significant increase of VEGF expression in the $\mathrm{O}_{2}+$ PTX group as compared with the $\mathrm{O}_{2}+\mathrm{NS}$ group. $n=7 /$ group. ${ }^{+} P<0.05$. (c) Immunofluorescence staining with an anti-vWF antibody. As compared with RA + NS lungs, the number of VWF-positive vessels (arrows) was significantly decreased in the $\mathrm{O}_{2}+$ NS group. PTX treatment increased pulmonary vascular density by $40 \%$ as compared with the $\mathrm{O}_{2}+$ NS group. $n=5 /$ group. ${ }^{*} P<$ 0.001 as compared with the RA + NS group; ${ }^{\dagger} P<0.01$ as compared with the $\mathrm{O}_{2}+\mathrm{NS}$ group. Original magnification: $\times 20$. Bar $=50 \mu \mathrm{m}$. NS, normal saline; $\mathrm{O}_{2}$, oxygen; $\mathrm{PTX}$, pentoxifylline; $\mathrm{RA}$, room air; VEGF, vascular endothelial growth factor; vWF, von Willebrand factor.

increasing AOE activity, upregulating VEGF, and decreasing pulmonary edema during oxygen exposure, show that PTX may be sufficient to improve survival but inadequate to blunt the effect of oxygen on alveolarization and fibrosis. However, other unexplored factors might have also accounted for the improved survival of newborn rats treated with PTX, including reduction in activation of nuclear factor- $\kappa \beta$ and production of tumor necrosis factor- $\alpha$, leading to attenuated lung injury (35), and the release of endothelium-derived nitric oxide by PTX in the pulmonary vascular bed, resulting in improved oxygenation (36).

There are several potential limitations of this study. First, different routes of administration, dosage, and length of treatment from hours to weeks for PTX have been used in numerous studies. The twice-a-day dosing used in this experiment might have not been optimal to exert all the potential benefits of PTX in the lungs of the newborn. However, this dosing was already found to be safe in preterm rats and beneficial in HILI as reported by ter Horst et al. (21). Second, the animals most susceptible to oxygen-induced injury were not analyzed due to their death. Whether PTX treatment during hyperoxia would have had similar effects on lungs of the pups that died remains unknown.

In summary, we found that PTX increases AOE activities, upregulates VEGF expression, improves lung vascularization, and decreases pulmonary edema and macrophage infiltration in newborn rats with hyperoxic lung injury. These enzymatic and molecular changes may ultimately have led to the improved survival during $9 \mathrm{~d}$ of hyperoxia. Further studies are needed to determine other mechanisms of PTX in lung protection as well as the most appropriate dose to obtain the maximal response. Nonetheless, these findings suggest that PTX therapy may play a role in the reduction of some of the features of BPD in premature infants.

\section{METHODS}

Animal Model and Experimental Protocol

Timed pregnant Sprague-Dawley rats were obtained from Charles River (Portage, MI). Pups were delivered naturally at term gestation, pooled, and randomly assigned to three groups to receive RA plus placebo, NS (RA + NS); hyperoxia $\left(>95 \% \mathrm{O}_{2}\right)$ plus $\mathrm{NS}\left(\mathrm{O}_{2}+\mathrm{NS}\right)$; or hyperoxia plus PTX $\left(\mathrm{O}_{2}+\right.$ PTX; Aventis Pharma, Mexico City, Mexico). Hyperoxia was achieved in a sealed Plexiglas chamber as previously described (31). PTX $(75 \mathrm{mg} / \mathrm{kg}$ equivalent to $3.75 \mathrm{ml} / \mathrm{kg}$ ) based on the efficacy and safety data previously reported by ter Horst et al. (21) or NS (same volume) was given via subcutaneous injection before $\mathrm{O}_{2}$ exposure and then twice a day during continuous exposure to RA or $\mathrm{O}_{2}$ for 4-9 d. Dams were rotated daily between $\mathrm{RA}$ and $\mathrm{O}_{2}$ to avoid oxygen toxicity. Animals were killed on day 4 and 9 with intraperitoneal injections of Eutasol (0.15 ml/kg; Virbac AH, Fort Worth, TX) for subsequent studies. The research protocol and procedures were reviewed and approved by the Animal Care and Use Committee at the University of Miami.

For biochemical assays and molecular analyses, lungs were perfused with ice-cold NS via the right ventricle until white. The perfused lungs were then excised, trimmed of extraparenchymal tracheal-bronchial and vascular tissue, weighed, frozen in liquid nitrogen, and stored in a $-80^{\circ} \mathrm{C}$ freezer. For histological and morphometric analyses, lungs were infused with $4 \%$ paraformaldehyde in phosphate-buffered saline via a tracheal catheter under $20 \mathrm{~cm} \mathrm{H} \mathrm{H}_{2} \mathrm{O}$ pressure for $5 \mathrm{~min}$ and then fixed in $4 \%$ paraformaldehyde overnight at $4{ }^{\circ} \mathrm{C}$. Dehydrated lung tissues were paraffin-embedded and 5 - $\mu \mathrm{m}$ tissue sections were prepared.

Unperfused lungs were excised en bloc and dissected away from the heart and thymus. The right upper lobe was immediately removed, blotted dry, and weighed immediately after removal. The lungs were then dried for $4 \mathrm{~d}$ in an oven at $60^{\circ} \mathrm{C}$ and reweighed. The wet-to-dryweight ratio was then calculated. 
Lung tissue sections were immunostained for Mac3, a macrophagespecific marker (BD Biosciences, San Jose, CA). The number of Mac3positive cells in the alveolar airspaces was counted from 10 random images taken with the $\times 40$ objective on each slide.

Frozen lung tissue was pooled in groups from two rat pups and homogenized in ice-cold phosphate-buffered saline. The lung homogenates were assayed by standard spectrophotometric techniques for activities of total SOD with the xanthine/xanthine oxidase method (37), of CAT using the rate of reduction of hydrogen peroxide (38), and of GPX using the rate of oxidation of nicotinamide adenine dinucleotide phosphate using cumene hydroxyperoxide as substrate (39). Lung homogenates were also assayed for total DNA content according to the Schmit-Thannhauser-Schneider method (40). Purified reference standards for all these assays were obtained commercially (Sigma Chemical, St Louis, MO).

Alveolarization was assessed on hematoxylin-eosin stained tissue sections by radial alveolar count and by mean linear intercept as previously described $(41,42)$.

To assess vascular development, immunofluorescence staining for von Willebrand factor (Dako, Carpinteria, CA), an endothelial-specific marker, was performed. Ten random images were taken with the $\times 20$ objective on each von Willebrand factor-stained slide. The vascular density was expressed as the average number of von Willebrand factor-positive vessels $(15-50 \mu \mathrm{m})$ counted per high-power field as previously described (42).

\section{RNA Isolation and Semiquantitative Reverse-Transcriptase PCR of VEGF Splice Variants}

Total RNA isolation and cDNA reverse transcription were performed as previously described (42). Reverse-transcriptase PCR was performed using a pair of rat VEGF primers: sense, 5'-CCAGCACATAGGAGAGATGAGCTTC-3' and antisense, 5'-GG TGTGGTGGTGACATGGTTAATC-3', which resulted in three bands $(262,394$, and $466 \mathrm{bp})$ corresponding to the three principal VEGF isoforms VEGF121, VEGF165, and VEGF189, respectively, expressed in rats (43). As a control, reverse-transcriptase PCR was also performed with $\beta$-actin-specific primers. The amplified cDNA fragments were then separated on $2 \%$ agarose gels and visualized by ethidium bromide staining. The intensity of the cDNA products was determined with the Quantity One Imaging Analysis Program (BioRad, Hercules, CA). The relative mRNA levels of each VEGF splicing variant were determined after normalization to $\beta$-actin.

\section{Measurement of VEGF Concentration and Western Blot Analysis}

Total VEGF protein concentration in lung homogenates was analyzed using a commercial enzyme-linked immunosorbent assay kit according to the manufacturer's instructions (R\&D Systems, Minneapolis, $\mathrm{MN})$. Total protein isolation and western blot analysis were performed as previously described (42).

\section{Statistical Analysis}

Data are expressed as mean \pm SD. Comparison among the groups was performed by ANOVA followed by the Holm-Sidak method as a post hoc analysis. For comparison of survival curves, Kaplan-Meier analysis followed by a log-rank test was used. A $P<0.05$ was considered significant.

\section{STATEMENT OF FINANCIAL DISCLOSURE}

This study was supported by Forest Pharmaceuticals and INO Therapeutics, through the Advancing Newborn Medicine Fellowship Grant Program and Project Newborn from the University of Miami.

\section{REFERENCES}

1. Jobe AH, Bancalari E. Bronchopulmonary dysplasia. Am J Respir Crit Care Med 2001;163:1723-9.

2. Bucher JR, Roberts RJ. The development of the newborn rat lung in hyperoxia: a dose-response study of lung growth, maturation, and changes in antioxidant enzyme activities. Pediatr Res 1981;15:999-1008.
3. Koo HC, Davis JM, Li Y, et al. Effects of transgene expression of superoxide dismutase and glutathione peroxidase on pulmonary epithelial cell growth in hyperoxia. Am J Physiol Lung Cell Mol Physiol 2005; 288:L718-26.

4. Wilborn AM, Evers LB, Canada AT. Oxygen toxicity to the developing lung of the mouse: role of reactive oxygen species. Pediatr Res 1996;40:225-32.

5. Yam J, Frank L, Roberts RJ. Oxygen toxicity: comparison of lung biochemical responses in neonatal and adult rats. Pediatr Res 1978;12:115-9.

6. Frank L, Groseclose EE. Preparation for birth into an $\mathrm{O}_{2}$-rich environment: the antioxidant enzymes in the developing rabbit lung. Pediatr Res 1984;18:240-4.

7. Gerdin E, Tydén O, Eriksson UJ. The development of antioxidant enzymatic defense in the perinatal rat lung: activities of superoxide dismutase, glutathione peroxidase, and catalase. Pediatr Res 1985;19:687-91.

8. Frank L, Sosenko IR. Failure of premature rabbits to increase antioxidant enzymes during hyperoxic exposure: increased susceptibility to pulmonary oxygen toxicity compared with term rabbits. Pediatr Res 1991;29:292-6.

9. Maniscalco WM, Watkins RH, D’Angio CT, Ryan RM. Hyperoxic injury decreases alveolar epithelial cell expression of vascular endothelial growth factor (VEGF) in neonatal rabbit lung. Am J Respir Cell Mol Biol 1997;16:557-67.

10. Thébaud B, Abman SH. Bronchopulmonary dysplasia: where have all the vessels gone? Roles of angiogenic growth factors in chronic lung disease. Am J Respir Crit Care Med 2007;175:978-85.

11. Watkins RH, D’Angio CT, Ryan RM, Patel A, Maniscalco WM. Differential expression of VEGF mRNA splice variants in newborn and adult hyperoxic lung injury. Am J Physiol 1999;276:L858-67.

12. Hsia CCW, Berberich MA, Driscoll B, et al. American Thoracic Society Documents. Mechanisms and limits of induced postnatal lung growth. Am J Respir Crit Care Med 2004;170:319-43.

13. Kunig AM, Balasubramaniam V, Markham NE, Seedorf G, Gien J, Abman SH. Recombinant human VEGF treatment transiently increases lung edema but enhances lung structure after neonatal hyperoxia. Am J Physiol Lung Cell Mol Physiol 2006;291:L1068-78.

14. Harris E, Schulzke SM, Patole SK. Pentoxifylline in preterm neonates: a systematic review. Paediatr Drugs 2010;12:301-11.

15. Michetti C, Coimbra R, Hoyt DB, Loomis W, Junger W, Wolf P. Pentoxifylline reduces acute lung injury in chronic endotoxemia. J Surg Res 2003;115:92-9.

16. Yada-Langoi MM, Macae M, Coimbra R, et al. Hypertonic saline and pentoxifylline prevent lung injury and bacterial translocation after hemorrhagic shock. Shock 2000;14:594-8.

17. Savas S, Delibas N, Savas C, Sütçü R, Cindas A. Pentoxifylline reduces biochemical markers of ischemia-reperfusion induced spinal cord injury in rabbits. Spinal Cord 2002;40:224-9.

18. Smalling WE Jr, Suguihara C, Huang J, Rodriguez MM, Bancalari E. Protective effect of pentoxifylline on volume-induced lung injury in newborn piglets. Biol Neonate 2004;86:15-21.

19. Korhonen K, Kiuru A, Svedström E, Kääpä P. Pentoxifylline reduces regional inflammatory and ventilatory disturbances in meconium-exposed piglet lungs. Pediatr Res 2004;56:901-6.

20. Lindsey HJ, Kisala JM, Ayala A, Lehman D, Herdon CD, Chaudry IH. Pentoxifylline attenuates oxygen-induced lung injury. J Surg Res 1994;56:543-8.

21. ter Horst SA, Wagenaar GT, de Boer E, et al. Pentoxifylline reduces fibrin deposition and prolongs survival in neonatal hyperoxic lung injury. J Appl Physiol 2004;97:2014-9.

22. Davis JM. Superoxide dismutase: a role in the prevention of chronic lung disease. Biol Neonate 1998;74:Suppl 1:29-34.

23. Tanswell AK, Freeman BA. Pulmonary antioxidant enzyme maturation in the fetal and neonatal rat. I. Developmental profiles. Pediatr Res 1984;18:584-7.

24. Ilizarov AM, Koo HC, Kazzaz JA, et al. Overexpression of manganese superoxide dismutase protects lung epithelial cells against oxidant injury. Am J Respir Cell Mol Biol 2001;24:436-41. 
25. Jakkula M, Le Cras TD, Gebb S, et al. Inhibition of angiogenesis decreases alveolarization in the developing rat lung. Am J Physiol Lung Cell Mol Physiol 2000;279:L600-7.

26. Bhatt AJ, Pryhuber GS, Huyck H, Watkins RH, Metlay LA, Maniscalco WM. Disrupted pulmonary vasculature and decreased vascular endothelial growth factor, Flt-1, and TIE-2 in human infants dying with bronchopulmonary dysplasia. Am J Respir Crit Care Med 2001;164:1971-80.

27. Maniscalco WM, Watkins RH, Pryhuber GS, Bhatt A, Shea C, Huyck H. Angiogenic factors and alveolar vasculature: development and alterations by injury in very premature baboons. Am J Physiol Lung Cell Mol Physiol 2002;282:L811-23.

28. Galambos C, Ng YS, Ali A, et al. Defective pulmonary development in the absence of heparin-binding vascular endothelial growth factor isoforms. Am J Respir Cell Mol Biol 2002; 27:194-203.

29. Greenberg JM, Thompson FY, Brooks SK, et al. Mesenchymal expression of vascular endothelial growth factors $\mathrm{d}$ and A defines vascular patterning in developing lung. Dev Dyn 2002;224:144-53.

30. Chen CM, Wang LF, Chou HC, Lang YD, Lai YP. Up-regulation of connective tissue growth factor in hyperoxia-induced lung fibrosis. Pediatr Res 2007;62:128-33.

31. Alapati D, Rong M, Chen S, et al. Connective tissue growth factor antibody therapy attenuates hyperoxia-induced lung injury in neonatal rats. Am J Respir Cell Mol Biol 2011;45:1169-77.

32. Lin SL, Chen RH, Chen YM, et al. Pentoxifylline attenuates tubulointerstitial fibrosis by blocking Smad3/4-activated transcription and profibrogenic effects of connective tissue growth factor. J Am Soc Nephrol 2005;16: 2702-13.

33. Raetsch C, Jia JD, Boigk G, et al. Pentoxifylline downregulates profibrogenic cytokines and procollagen I expression in rat secondary biliary fibrosis. Gut 2002;50:241-7.
34. Yang S, Zhou M, Koo DJ, Chaudry IH, Wang P. Pentoxifylline prevents the transition from the hyperdynamic to hypodynamic response during sepsis. Am J Physiol 1999;277:H1036-44.

35. Coimbra R, Melbostad H, Loomis W, et al. LPS-induced acute lung injury is attenuated by phosphodiesterase inhibition: effects on proinflammatory mediators, metalloproteinases, NF-kappaB, and ICAM-1 expression. J Trauma 2006;60:115-25.

36. Kaye AD, Ibrahim IN, Kadowitz PJ, Nossaman BD. Analysis of responses to pentoxifylline in the pulmonary vascular bed of the cat. Crit Care Med 1996;24:263-7.

37. McCord JM, Fridovich I. Superoxide dismutase. An enzymic function for erythrocuprein (hemocuprein). J Biol Chem 1969;244:6049-55.

38. Holmes RS, Masters CJ. Epigenetic interconversions of the multiple forms of mouse liver catalase. FEBS Lett 1970;11:45-8.

39. Paglia DE, Valentine WN. Studies on the quantitative and qualitative characterization of erythrocyte glutathione peroxidase. J Lab Clin Med 1967;70:158-69.

40 Schmidt G. Determination of nucleic acids by phosphorus analysis. In: Colowick SP, Kaplan NO, eds. Methods in Enzymology, vol. 3. New York: Academic Press, 1957:671-9.

41. Cooney TP, Thurlbeck WM. The radial alveolar count method of Emery and Mithal: a reappraisal 1-postnatal lung growth. Thorax 1982;37: 572-9.

42. Chen S, Rong M, Platteau A, et al. CTGF disrupts alveolarization and induces pulmonary hypertension in neonatal mice: implication in the pathogenesis of severe bronchopulmonary dysplasia. Am J Physiol Lung Cell Mol Physiol 2011;300:L330-40.

43. Zheng W, Seftor EA, Meininger CJ, Hendrix MJ, Tomanek RJ. Mechanisms of coronary angiogenesis in response to stretch: role of VEGF and TGF- $\beta$. Am J Physiol Heart Circ Physiol 2001;280:H909-17. 